\title{
An Averaging Model for Chaotic System with Periodic Time-Varying Parameter
}

\author{
Yu Mao ${ }^{\mathrm{a}}$, Wallace K.S. Tang ${ }^{\mathrm{a}}$, Marius-F. Danca ${ }^{\mathrm{b}, \mathrm{c}}$ \\ ${ }^{a}$ Department of Electronic Engineering, City University of Hong Kong, Hong Kong \\ ${ }^{b}$ Department of Mathematics and Computer Science, Avram Iancu University, Cluj-Napoca, Romania \\ ${ }^{c}$ Romanian Institute of Science and Technology, Cluj-Napoca, Romania
}

\begin{abstract}
In this paper, we provide a mathematical justification to explain the dynamics of chaotic system with periodic time-varying parameter which have been illustrated by some of us in a previous paper [1]. Based on an equivalent averaging model, it is proved that such a parametric time-varying system follows the same trajectory of its averaging model, provided that the parameter is varied periodically with a sufficiently high frequency. Some other observations related with this class of chaotic systems are also remarked in this paper.

Keywords: Averaging model, Chaotic system, Chen system, Chua's circuit, Parameter switching, Time-varying system
\end{abstract}

\section{Introduction}

System with parameter variations have aroused a lot of intersets, not only because of its importance in applications, but also due to its attractive complex dynamics for theoretical analysis. Many studies have been reported for the stability issues of switched linear and nonlinear systems $[2,3,4]$. The uses of switching dynamics have also been explored, such as, to generate complex attractors by switching system parameters [1], to achieve synchronization and control [5], to name a few. Recently, similar studies have also been extended to complex networks, for which time-varying topologies are in concern $[6,7]$.

In this paper, we are interested in studying analytically the behaviour of a chaotic system with periodic time-varying parameter. Extensive simulations have been carried out in $[1,8,9]$, showing that such a system acts similarly as its corresponding averaging model. In $[1,9]$ it is illustrated with numerical simulations that attractors of a chaotic system can be synthesized by switching a parameter periodically. A similar switching algorithm was used in [8] where a random switching rule has been applied.

Our analysis is mainly carried out based on the averaging theory [10]. In classical averaging theory, a system is assumed to be expressed as $\dot{x}=\lambda f(t, x)$, where $\lambda$ is a small parameter representing the term of "slowness" governing the time scale of slow evolution of system parameters, and $f$ is n-dimensional and T-periodic (or is a KBM-vector field) [10]. It is found that its solution and that of the corresponding average model, taking the form $\dot{x}=\lambda f^{0}(x)$, with $f^{0}(x)$ is the average value of $f(x, t)$, present a difference of 
$O(\lambda)$. This averaging principle has also been extended to partial differenital equations, integro-differential equations, infinite-dimensional system, time delay systems and so on (see examples in $[4,11,12,13]$ ).

However, chaotic systems cannot be reformulated in the standard form or its variants, as the function is not periodic but the parameter is. Hence, it is the objective of this paper to establish a theory to prove analytically that chaotic systems with periodically varying parameter remains close enough to the trajectory that evolves from its equivalent average model, provided that the period of the change of parameter is sufficiently small.

The organization of this paper is as follows. In Sect. 2, the main theorem is given and proved. Simulations for some typical chaotic systems, including Chua's circuit and Chen system are then presented in Sect. 3, demonstrating the effect of parametric oscillation and some particular remarks are given. Finally, conclusions are drawn in Sect. 4.

\section{Average Model for Nonlinear System with Periodic Time-Varying Param- eter}

Consider a class of nonlinear continuous-time and dissipative systems given in the following form:

$$
\dot{\mathbf{x}}(t)=f(\mathbf{x}(t))+p(t / \lambda) \mathbf{B} \mathbf{x}(t), \quad \mathbf{x}(0)=x_{0},
$$

where $\mathbf{x} \in \Re^{n}$ is the state vector; $f: \Re^{n} \rightarrow \Re^{n}$ is a sufficiently smooth vector field on $\Re^{n}$ to assure the existence and uniqueness of solution; $t \in I=[0, \infty) ; \mathbf{B} \in \Re^{n \times n}$ is a constant matrix; and $p: I \rightarrow R$ is a periodic function of period $T$ having a mean value of $q$, i.e.

$$
\frac{1}{T} \int_{t}^{t+T} p(u) d u=q \quad \forall t \in I .
$$

and define an average model of (1) expressed as follows:

$$
\dot{\mathbf{y}}(t)=f(\mathbf{y}(t))+q \mathbf{B y}(t), \quad \mathbf{y}(0)=\mathbf{y}_{0},
$$

where $\mathbf{y} \in \Re^{n}$ is the state vector.

Supposing that (3) admits $\mathbf{s}(t)$ as the unique solution, by linearizing (3) on a neighborhood of $\mathbf{s}(t)$, one obtains the following initial value problem:

$$
\dot{\varepsilon}(t)=[F(t)+q \mathbf{B}] \varepsilon(t)=A_{q}(t) \varepsilon(t), \quad \varepsilon(0)=\varepsilon_{0},
$$

where $\varepsilon(t)=\mathbf{y}(t)-\mathbf{s}(t) ; A_{q}(t)=F(t)+q \mathbf{B}$; and $F(t)$ denotes the Jacobian of $f$ evaluated at $\mathbf{s}(t)$.

Since $\mathbf{s}(t)$ is the solution of $(3), \varepsilon(t)=0$ for $t \in I$ should be the solution of (4). Denoting $\Gamma_{s}$ as the domain of attraction of $\varepsilon=0$,

$$
\Gamma_{s}:=\left\{\varepsilon_{0} \in \Re^{n}: \lim _{t \rightarrow \infty} \varepsilon(t)=0\right\},
$$

and considering the below dynamical system obtained by linearizing (1) with $\mathbf{e}(t)=$ $\mathbf{x}(t)-\mathbf{s}(t)$ and $\mathbf{x} \in \Gamma_{s}$,

$$
\dot{\mathbf{e}}(t)=[F(t)+p(t / \lambda) \mathbf{B}] \mathbf{e}(t)=A_{p}(t) \mathbf{e}(t), \quad \mathbf{e}(0)=\mathbf{e}_{0},
$$

where $A_{p}(t)=F(t)+p(t / \lambda) \mathbf{B}$, the following theorem can be established. 
Theorem 1. Assuming that Eq. (4) is uniformly exponentially stable, i.e.

$$
\exists C>0, \mu>0 \quad \text { such that } \varepsilon(t) \leq C\left\|\varepsilon_{0}\right\| \exp (-\mu t),
$$

with $\mathbf{e}_{0}=\varepsilon_{0}$, there exists a positive scalar $\lambda>0$, such that $\lim _{t \rightarrow \infty}\|\mathbf{e}(t)-\varepsilon(t)\|=\delta\left(\lambda^{2}\right)$, where $\delta\left(\lambda^{2}\right)$ is an order function*.

Proof: Due to the uniqueness of solution of (1), it follows that the initial value problems (4) and (6) have the unique solutions $\varepsilon(t)=\Phi_{A_{q}}(t, 0) \varepsilon_{0}$ and $\mathbf{e}(t)=\Phi_{A_{p}}(t, 0) \mathbf{e}_{0}$, respectively, where $\Phi_{A_{q}}(t, 0)$ and $\Phi_{A_{p}}(t, 0)$ are the corresponding transition matrices and $\Phi_{A}(t, 0)=\Phi_{A}(t, \tau) \Phi_{A}(\tau, 0)$ for $t \geq \tau \geq 0$.

Remark 1. The existence of $\Phi_{A_{p}}(t, 0)$ and $\Phi_{A_{q}}(t, 0)$ is based on the existence and uniqueness of the solutions of (6) and (4), respectively. See Theorem 3.2 in [14].

Let's partition the existence interval $I=[0, \lambda T] \bigcup[\lambda T, 2 \lambda T] \cdots$, and let $\varepsilon_{k}(t)$ be the solution of Eq. (4) in the subinterval $I_{k}=[k \lambda T,(k+1) \lambda T]$. We will prove that

$$
\left\|\mathbf{e}((k+1) \lambda T)-\varepsilon_{k}((k+1) \lambda T)\right\| \leq \delta\left(\lambda^{2}\right)
$$

if the initial condition at $t=k \lambda T$ is chosen as $\varepsilon_{k}(k \lambda T)=\mathbf{e}(k \lambda T)$.

As explained in [15], a transition matrix can be approximated by a generalized PeanoBaker series. Therefore,

$$
\begin{gathered}
\Phi_{A}(t, \tau)=I_{n}+\int_{\tau}^{t} A\left(s_{1}\right) d s_{1}+\sum_{i=2}^{\infty}\left[\int_{\tau}^{t} A\left(s_{1}\right) \int_{\tau}^{s_{1}} A\left(s_{2}\right)\right. \\
\left.\ldots \int_{\tau}^{s_{i-1}} A\left(s_{i}\right) d s_{i} \cdots d s_{2} d s_{1}\right]
\end{gathered}
$$

where $t \geq \tau \geq 0$ and $I_{n}$ is the identity matrix of size $n$.

On each subinterval $I_{k}$, denoting $H_{k}=\Phi_{A_{p}}((k+1) \lambda T, k \lambda T)-\Phi_{A_{q}}((k+1) \lambda T, k \lambda T)$, one has

$$
\begin{gathered}
H_{k}=I_{n}+\int_{k \lambda T}^{(k+1) \lambda T} A_{p}\left(s_{1}\right) d s_{1} \sum_{i=2}^{\infty}\left[\int_{k \lambda T}^{(k+1) \lambda T} A_{p}\left(s_{1}\right) \int_{k \lambda T}^{s_{1}} A_{p}\left(s_{2}\right)\right. \\
\left.\ldots \int_{k \lambda T}^{s_{i-1}} A_{p}\left(s_{i}\right) d s_{i} \cdots d s_{2} d s_{1}\right] \\
-\left\{I_{n}+\int_{k \lambda T}^{(k+1) \lambda T} A_{q}\left(s_{1}\right) d s_{1}+\sum_{i=2}^{\infty}\left[\int_{k \lambda T}^{(k+1) \lambda T} A_{q}\left(s_{1}\right) \int_{k \lambda T}^{s_{1}} A_{q}\left(s_{2}\right)\right.\right. \\
\left.\left.\ldots \int_{k \lambda T}^{s_{i-1}} A_{q}\left(s_{i}\right) d s_{i} \cdots d s_{2} d s_{1}\right]\right\}
\end{gathered}
$$

*An order function $\delta\left(\lambda^{2}\right)$ (see [10] p.11), implies that there exists $m$ s.t. $\left|\delta\left(\lambda^{2}\right)\right| \leq m \lambda^{2}$ when $\lambda$ is sufficiently small. 
Using the periodic property given in (2),

$$
\begin{aligned}
\int_{k \lambda T}^{(k+1) \lambda T} A_{p}\left(s_{1}\right) d s_{1} & =\int_{k \lambda T}^{(k+1) \lambda T} F\left(s_{1}\right)+p\left(s_{1} / \lambda\right) B d s_{1} \\
& =\int_{k \lambda T}^{(k+1) \lambda T} F\left(s_{1}\right) d s_{1}+\lambda T q B \\
& =\int_{k \lambda T}^{(k+1) \lambda T}\left[F\left(s_{1}\right)+q B\right] d s_{1} \\
& =\int_{k \lambda T}^{(k+1) \lambda T} A_{q}\left(s_{1}\right) d s_{1},
\end{aligned}
$$

and (10) becomes

$$
\begin{aligned}
H_{k}= & \sum_{i=2}^{\infty}\left[\int_{k \lambda T}^{(k+1) \lambda T} A_{p}\left(s_{1}\right) \int_{k \lambda T}^{s_{1}} A_{p}\left(s_{2}\right) \ldots \int_{k \lambda T}^{s_{i-1}} A_{p}\left(s_{i}\right) d s_{i} \cdots d s_{2} d s_{1}\right. \\
& \left.-\int_{k \lambda T}^{(k+1) \lambda T} A_{q}\left(s_{1}\right) \int_{k \lambda T}^{s_{1}} A_{q}\left(s_{2}\right) \ldots \int_{k \lambda T}^{s_{i-1}} A_{q}\left(s_{i}\right) d s_{i} \cdots d s_{2} d s_{1}\right] .
\end{aligned}
$$

Defining $\alpha_{k} \equiv \sup \max \left(\left\|A_{p}\right\|,\left\|A_{q}\right\|\right)^{\dagger}$, the bound for $H_{k}$ can be computed as follows:

$$
\begin{aligned}
\left\|H_{k}\right\|=\| \sum_{i=2}^{\infty} & {\left[\int_{k \lambda T}^{(k+1) \lambda T} A_{p}\left(s_{1}\right) \int_{k \lambda T}^{s_{1}} A_{p}\left(s_{2}\right) \ldots \int_{k \lambda T}^{s_{i-1}} A_{p}\left(s_{i}\right) d s_{i} \cdots d s_{2} d s_{1}\right.} \\
& \left.\quad-\int_{k \lambda T}^{(k+1) \lambda T} A_{q}\left(s_{1}\right) \int_{k \lambda T}^{s_{1}} A_{q}\left(s_{2}\right) \ldots \int_{k \lambda T}^{s_{i-1}} A_{q}\left(s_{i}\right) d s_{i} \cdots d s_{2} d s_{1}\right] \| \\
\leq & \sum_{i=2}^{\infty}\left[\left\|\int_{k \lambda T}^{(k+1) \lambda T} A_{p}\left(s_{1}\right) \int_{k \lambda T}^{s_{1}} A_{p}\left(s_{2}\right) \ldots \int_{k \lambda T}^{s_{i-1}} A_{p}\left(s_{i}\right) d s_{i} \cdots d s_{2} d s_{1}\right\|\right. \\
& \left.\quad+\left\|\int_{k \lambda T}^{(k+1) \lambda T} A_{q}\left(s_{1}\right) \int_{k \lambda T}^{s_{1}} A_{q}\left(s_{2}\right) \ldots \int_{k \lambda T}^{s_{i-1}} A_{q}\left(s_{i}\right) d s_{i} \cdots d s_{2} d s_{1}\right\|\right] \\
\leq & 2 \sum_{i=2}^{\infty} \int_{k \lambda T}^{(k+1) \lambda T} \alpha_{k} \int_{k \lambda T}^{s_{1}} \alpha_{n} \ldots \int_{k \lambda T}^{s_{i-1}} \alpha_{k} d s_{i} \cdots d s_{2} d s_{1} \\
= & 2 \sum_{i=2}^{\infty} \frac{\left(\alpha_{k} \lambda T\right)^{i}}{i !} .
\end{aligned}
$$

Therefore, using (13), one has

$$
\begin{aligned}
\left\|\mathbf{e}((k+1) \lambda T)-\varepsilon_{k}((k+1) \lambda T)\right\| & =\left\|H_{k}\right\|\left\|\varepsilon_{k}(k \lambda T)\right\| \\
& \leq 2 \sum_{i=2}^{\infty} \frac{\left(\alpha_{k} \lambda T\right)^{i}}{i !}\left\|\varepsilon_{k}(k \lambda T)\right\| \equiv \delta\left(\lambda^{2}\right) .
\end{aligned}
$$

${ }^{\dagger}$ The functions $A_{p}(t)$ and $A_{q}(t)$ are assumed to be bounded, i.e. $\left\|A_{p}(t)\right\| \leq a_{p}$ and $\left\|A_{q}(t)\right\| \leq a_{q}$, for some constants $a_{p}$ and $a_{q}$, and $\|\cdot\|$ is the Euclidean norm. 
Since (4) is uniformly exponentially stable for any two solutions, $\tilde{\varepsilon}(t)$ and $\hat{\varepsilon}(t)$, starting in $\Gamma_{s}$, by Gronwall's inequality and $(7)$, it can be derived that

$$
\|\tilde{\varepsilon}(t)-\hat{\varepsilon}(t)\| \leq C\left\|\tilde{\varepsilon}\left(t_{0}\right)-\hat{\varepsilon}\left(t_{0}\right)\right\| \exp \left(-\mu\left(t-t_{0}\right)\right) \quad \forall t \geq t_{0} .
$$

If $\left(t-t_{0}\right)$ is large enough,

$$
\|\tilde{\varepsilon}(t)-\hat{\varepsilon}(t)\| \leq L\left\|\tilde{\varepsilon}\left(t_{0}\right)-\hat{\varepsilon}\left(t_{0}\right)\right\|,
$$

where $0<L<1$.

Next, taking into account of the inequality (15) on $I_{k}$ with the initial condition at $k \lambda T$ and considering $\lambda T>\frac{1}{\mu} \ln C$, the following relationship is obtained

$$
\left\|\varepsilon((k+1) \lambda T)-\varepsilon_{k}((k+1) \lambda T)\right\| \leq L\left\|\varepsilon(k \lambda T)-\varepsilon_{k}(k \lambda T)\right\|,
$$

for $k=1,2, \cdots$.

By triangular inequality, one obtains

$$
\begin{aligned}
& \|\mathbf{e}((k+1) \lambda T)-\varepsilon((k+1) \lambda T)\| \\
\leq & \delta\left(\lambda^{2}\right)+L\left\|\varepsilon(k \lambda T)-\varepsilon_{k}(k \lambda T)\right\| \\
\leq & \delta\left(\lambda^{2}\right)+L\|\mathbf{e}(k \lambda T)-\varepsilon(k \lambda T)\|+L\left\|\mathbf{e}(k \lambda T)-\varepsilon_{k}(k \lambda T)\right\| \\
\leq & (1+L) \delta\left(\lambda^{2}\right)+L\|\mathbf{e}(k \lambda T)-\varepsilon(k \lambda T)\| .
\end{aligned}
$$

and by recursion,

$$
\|\mathbf{e}((k+1) \lambda T)-\varepsilon((k+1) \lambda T)\| \leq(1+L) \delta\left(\lambda^{2}\right)\left(1+L+L^{2}+\cdots+L^{k}\right) .
$$

Taking the limit $k \rightarrow \infty$, one has

$$
\lim _{t \rightarrow \infty}\|\mathbf{e}(t)-\varepsilon(t)\| \leq \frac{1+L}{1-L} \delta\left(\lambda^{2}\right),
$$

which completes the proof.

Remark 2. (i) Based on Theorem 1, it is possible to choose $\lambda$ small enough such that $\lim _{t \rightarrow \infty}\|\mathbf{e}(t)-\varepsilon(t)\| \leq L \lambda^{2}$. Consequently, the solution $x(t)$ in (1) will exhibit a similar behavior as the solution $y(t)$ of the average model (3). (ii) For practical reasons, if $T$ is set to be small enough, we can take $\lambda=1$ instead of a small $\lambda$.

\section{Simulation Results}

In this section, some special cases are considered to further illustrate the theorem presented in Sect. 2. In all the presented simulations, as explained in Remark 2 (ii), $\lambda$ is taken to be unity and $T$ is in the order of $10^{-2}$. 


\subsection{Chua's Circuit}

The first example is the famous Chua's circuit [16] given as below:

$$
\left\{\begin{array}{l}
\dot{x}=p[y-g(x)] \\
\dot{y}=x-y+z \\
\dot{z}=-b y
\end{array}\right.
$$

where $g(x)=m_{1} x+0.5\left(m_{0}-m_{1}\right)(|x+1|-|x-1|), b=14 \frac{2}{7}, m_{0}=-\frac{1}{7}, m_{1}=\frac{2}{7}$ and $p$ is the time varying parameter.

Figures 1 (a) and (b) depict the two periodic attractors when $p$ equals to 9.5 and 9.69 , respectively. Now, consider that $p=p_{1}(t)$ such that

$$
p_{1}(t)= \begin{cases}9.5 & 0 \leq t<\frac{k T}{2} \\ 9.6 & \frac{k T}{2} \leq t<k T\end{cases}
$$

where $k=1,2, \ldots$ and $T=0.01$.

Thus, $p_{1}(t)$ is a bistable function taking 9.5 and 9.69 equally in time with period $\mathrm{T}=0.01$. The mean value of $p_{1}$ computed by (2) over one period is $q_{1}=9.595$. The phase portrait obtained by such a switching system is given in Fig. 1 (c). For comparison, the corresponding phase portrait of an average model with $p$ replaced by the mean value $q_{1}=9.595$ is also shown in Fig. 1 (d).

Similarly, if we use another bistable function $p_{2}$ with the same period while $p$ takes 9.49 and 9.51 equally in time (the mean is $q_{2}=9.5$ ), a stable periodic attractor is observed even though these two values give chaotic attractors as shown in Fig. 2.

Based on our proof, more complex functions can be adopted and present no difference if they possess the same mean value. Two examples are shown in Fig. 3 (b) and (d) for which the mean values of $p_{3}$ and $p_{4}$ are both equal to 9.5 , and it can be easily observed that the obtained attractors in Fig. 3 (a) and (c) are well matched with that obtained in Fig. $2(\mathrm{~d})$.

\subsection{Chen System}

Our next example is the Chen system [17], expressed in the form of

$$
\left\{\begin{array}{l}
\dot{x}=-a x+p y \\
\dot{y}=(c-a) x-x z+c y \\
\dot{z}=x y-b z
\end{array}\right.
$$

where $a=35, b=3, c=28$, and $p$ is the time-varying parameter.

Based on the bifurcation of (22), it is known that chaotic attractors can be obtained as shown in Fig. 4 (a) and (b), respectively, when $p$ equals to 56 and 58 . Now let $p_{5}(t)$ be a bistable function with $\mathrm{T}=0.01$ taking its values between 56 and 58 equally in time, Fig. 4 (c) depicts the phase portrait obtained by a time-varying system when $p=p_{5}(t)$ in (22). The orbit is the same as that shown in Fig. 4 (d) when $p$ is replaced by the mean value $q_{5}=57$.

Remark 3. (i) In practical, $T$ will not be arbitrarily small. Therefore, a small difference can be observed between the attractors obtained by the parameter-varying system and its averaging model. For example, the attractors shown in Fig. 3 (b) and 4 (c) are not exactly 
identical to the attractors given in Fig. $2(d)$ and 4 (d) obtained by their averaging models.

(ii) In a chaotic system, mutliple stable attractors may coexist. Therefore, the small deviation may cause the parameter-varying system entering the $\Gamma_{s}$ of another stable attractor (expecially when the initial value is far away from the trajectory of the attractor, such as $x_{0}=10 ; y_{0}=9$ and $z_{0}=12$ in our example) and remain inside as shown in Fig. 5 (a) while the average system still rests on the same orbit (same as Fig. 4 (d)). This situation can be improved as shown in Fig. 5 (b), when $T$ is reduced and a smaller deviation is implied as derived in (19).

\section{Conclusion}

In this paper, an averaging theory is established to explain the asymptotic behaviour of chaotic systems with a periodically changing parameter. It is proved that such a parametric time-varying system will exhibit similar attractor of its equivalent average model, when the frequency is high enough. The phenomenon is also demonstrated with various typical chaotic systems and some special conditions are remarked.

\section{Acknowledgements}

The work described in this paper was fully supported by a grant from the Research Grants Council of the Hong Kong Special Administrative Region, China [Project No. CityU 120708].

\section{References}

[1] M.F. Danca, W.K.S. Tang, G. Chen, A switching scheme for synthesizing attractors of dissipative chaotic systems, Appl. Math. Comput. 201 (2008) 650-667 (2008).

[2] H. Lin, P.J. Antsaklis, Stability and stabilizability of switched linear systems: A survey of recent results, IEEE Trans. Auto. Cont. 54 (2009) 308-322.

[3] Z. Sun, A note on marginal stability of switched systems, IEEE Trans. Automatic Control. 53 (2008) 625-631.

[4] J. Tsinias, A. Stamati, Local asymptotic stability via averaging for time-varying systems with unbounded dynamics with respect to time, IEEE Trans. Auto. Cont. 54 (2009) 1374-1381.

[5] F. Fiorilli, M. Porfiri, Fast-switching pulse synchroniztaion of chaotic oscillators, Proc. 2008 Amer. Cont. Conf. (2008) 5092-5097.

[6] M. Hasler, I. Belykh, V.N. Belykh, Classes of stochastically switched (blinking) systems, Proc. Int. Symp. Circuits and Systems. (2007) 1665-1668.

[7] J. Zhao, D.J. Hill, T. Liu, Synchronization of complex dynamical networks with switching topology: A switched system point of view, Automatica. 45 (2009) 2502-2511.

[8] M.F. Danca, Random parameter-switching synthesis of a class of hyperbolic attractors, Chaos. 18:033111 (2008) 1-9.

[9] M.F. Danca, Finding stable attractors of a class of dissipative dynamical systems by numerical parameter switching, Dynamical Systems. DOI:10.1080/14689360903401278 (2009).

[10] J.A. Sanders, F. Verhulst, Averaging Methods in Nonlinear Dynamical Systems, Springer-Verlag, New York, 1985

[11] M. Lakrib, On the averaging method for differential equation with delay, Electronic J. Diff. Eq. 65 (2002) $1-16$

[12] B. Lehman, S.P.Weibel, Averaging theory for delay difference equations with time-varying delays, SIAM J. Applied Mathematics. 59 (1999) 1487-1506.

[13] J. Peuteman, D. Aeyels, Exponential stability of nonlinear time-varying differential equations and partial averaging, Math. Control Signals Systems. 15 (2002) 42-70 . 
[14] K.K. Hassan, Nonlinear Systems, Prentice Hall, New Jersey, 2002.

[15] J.J. Dacunha, Transition matrix and generalized matrix exponential via the Peano-Baker Series, J. Difference Eq. and Appl. 11 (2005) 1245-1264.

[16] L.O. Chua, M. Komuro, T. Matsumoto, The double scroll family, IEEE Trans. Circ. Syst. 33 (1986) $1072-1118$.

[17] G. Chen, T. Ueta, Yet another chaotic attractor, Int. J. Bifur. Chaos. 9 (1999) 1465-1466. 


\section{List of Figures}

$1 \quad$ Phase portraits ( $x$ versus $y$ ) of system (20) with (a) $p=9.5$; (b) $p=9.69$; (c) $p=p_{1}(t)$; (d) $p=q_{1}=9.595 \ldots \ldots \ldots \ldots \ldots \ldots$

$2 \quad$ Phase portrait ( $x$ versus $y$ ) of system (20) with (a) $p=9.49$; (b) $p=9.51$;

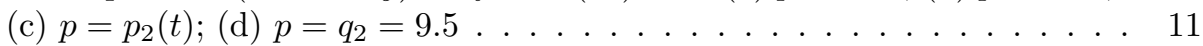

3 (a) $p_{3}$ taking the values of $9.52,9.48,9.51$ and 9.49 equally in time with $\mathrm{T}=0.01$; (b) phase portrait ( $x$ versus $y$ ) of system (20) with $p=p_{3}(t)$; (c) $p_{4}(t)=\sin (200 t)+\sin (400 t)+9.5$; (d) phase portrait $(x$ versus $y)$ of

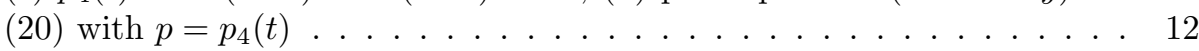

4 Phase portrait ( $x$ versus $z$ ) of system (22) with initial value of $x_{0}=1, y_{0}=$ $0.1, z_{0}=0.1$ and (a) $p=56$; (b) $p=58$; (c) $p=p_{5}(t)$; (d) $p=q_{5}=57 . \ldots 13$

5 Phase portrait ( $x$ versus $z$ ) of system (20) with initial value of $x_{0}=$ $10, y_{0}=9, z_{0}=12$ and $p=p_{5}(t)$ when (a) $T=0.01$ and (b) $T=0.005 . . \quad 14$ 


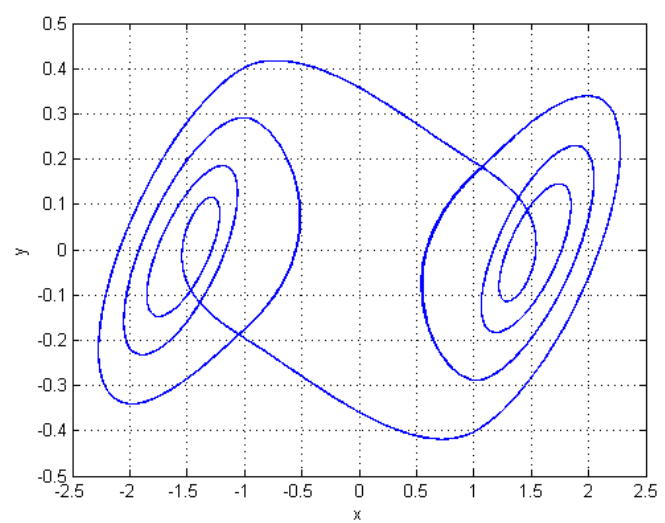

(a)

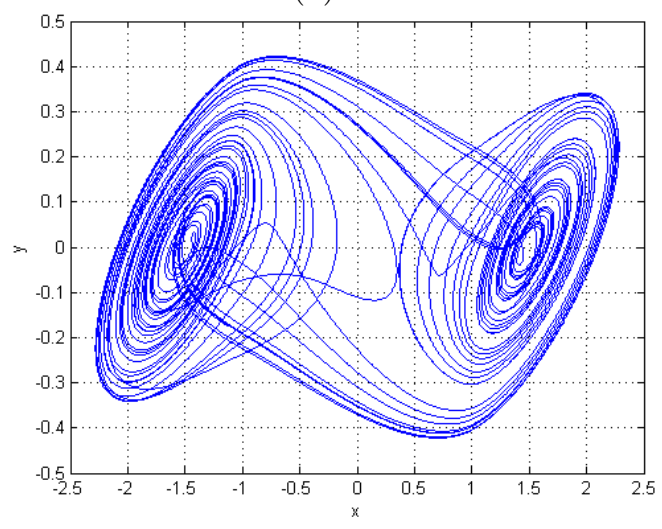

(c)

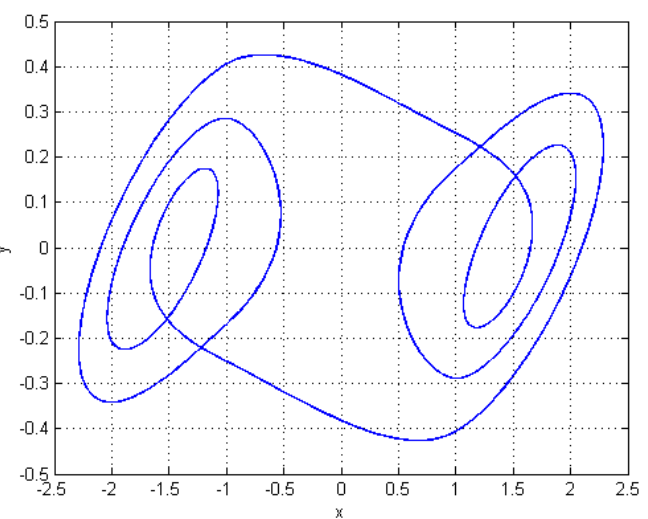

(b)

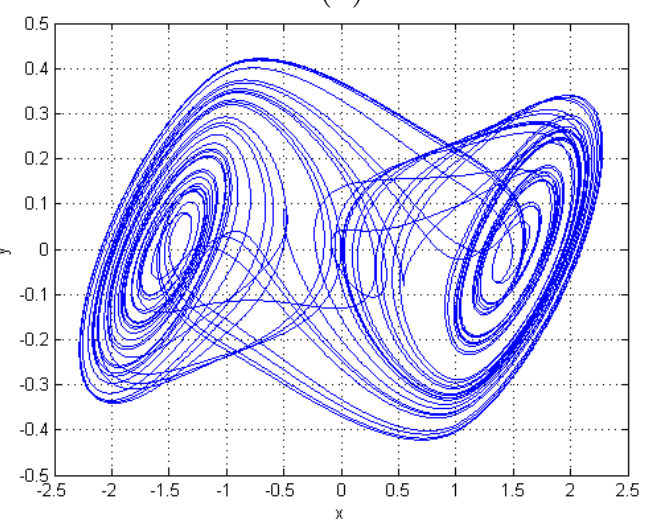

(d)

Figure 1: Phase portraits ( $x$ versus $y$ ) of system (20) with (a) $p=9.5$; (b) $p=9.69$; (c) $p=p_{1}(t)$; (d) $p=q_{1}=9.595$ 


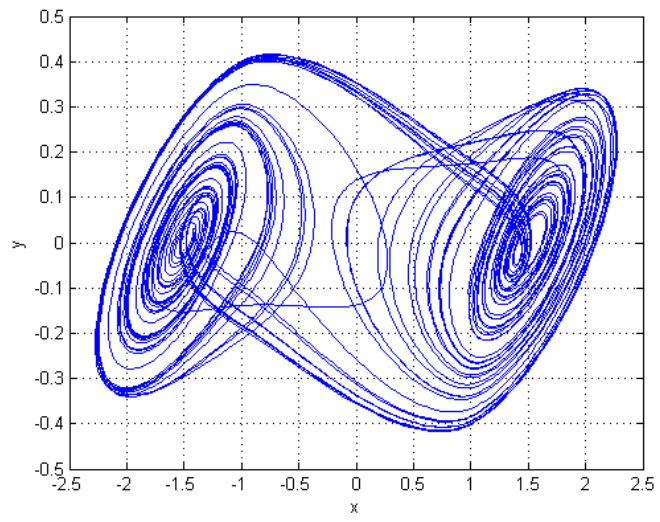

(a)

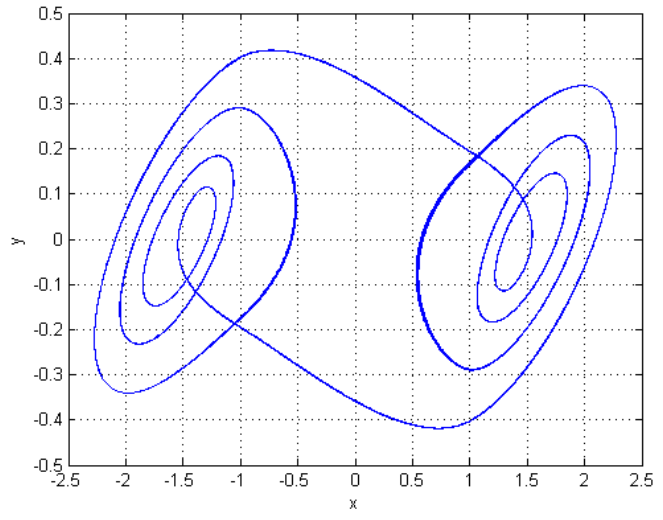

(c)

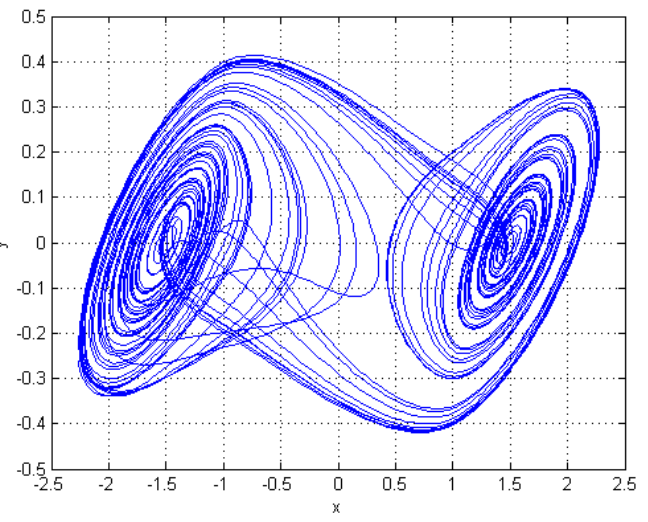

(b)

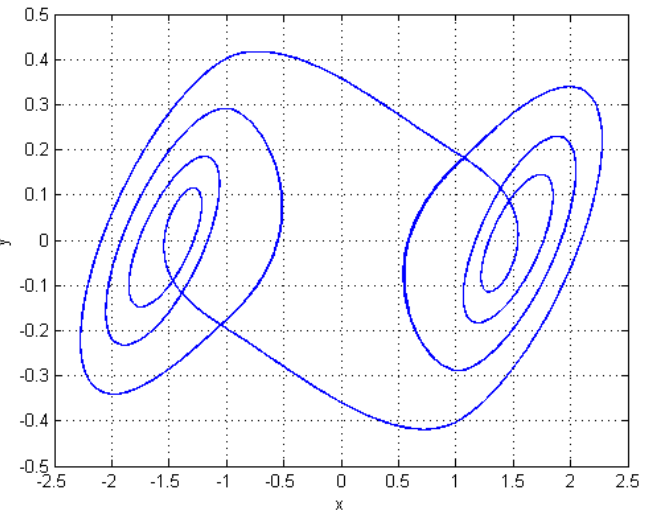

(d)

Figure 2: Phase portrait ( $x$ versus $y$ ) of system (20) with (a) $p=9.49$; (b) $p=9.51$; (c) $p=p_{2}(t)$; (d) $p=q_{2}=9.5$ 


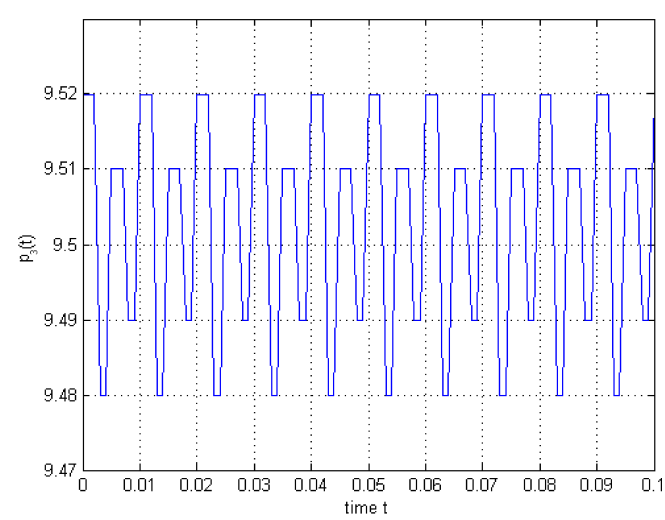

(a)

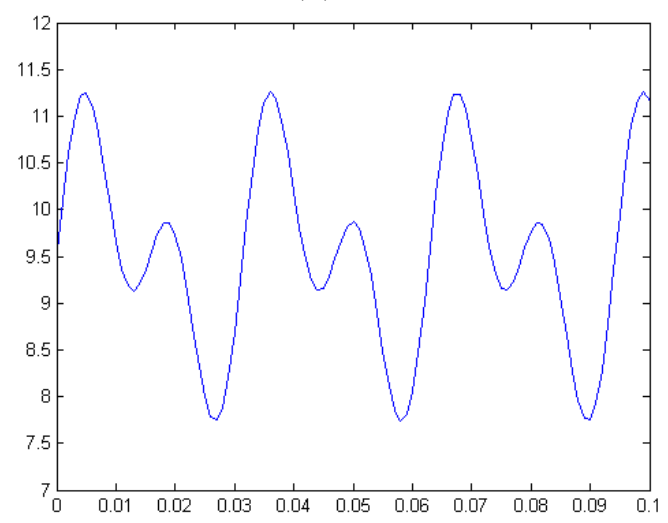

(c)

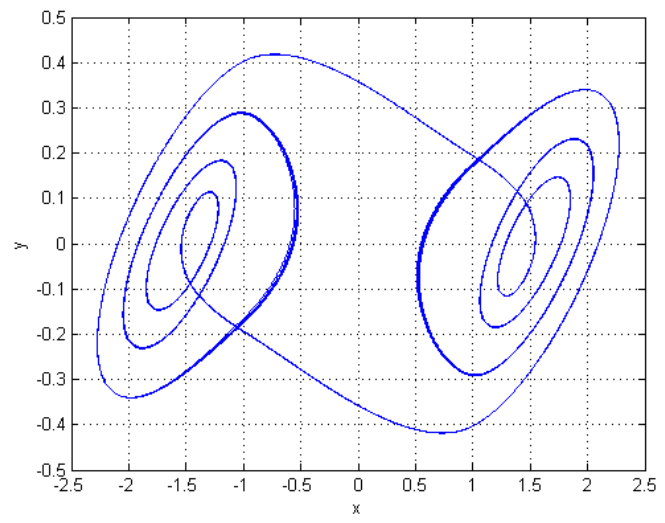

(b)

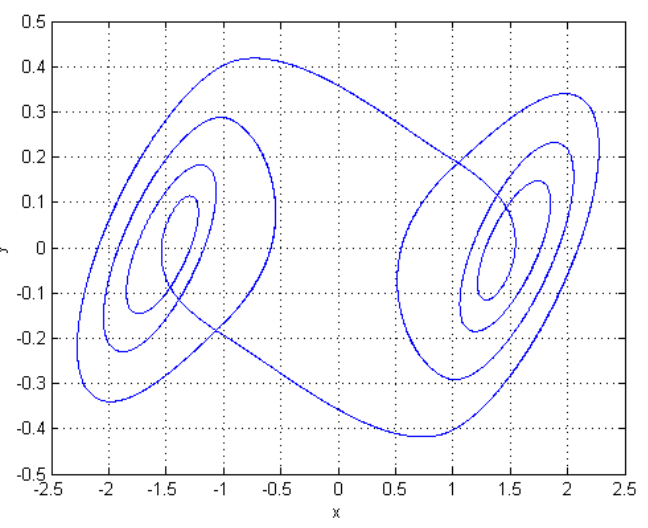

(d)

Figure 3: (a) $p_{3}$ taking the values of $9.52,9.48,9.51$ and 9.49 equally in time with $\mathrm{T}=0.01$; (b) phase portrait $(x$ versus $y)$ of system (20) with $p=p_{3}(t)$; (c) $p_{4}(t)=\sin (200 t)+\sin (400 t)+9.5$; (d) phase portrait $(x$ versus $y)$ of $(20)$ with $p=p_{4}(t)$ 


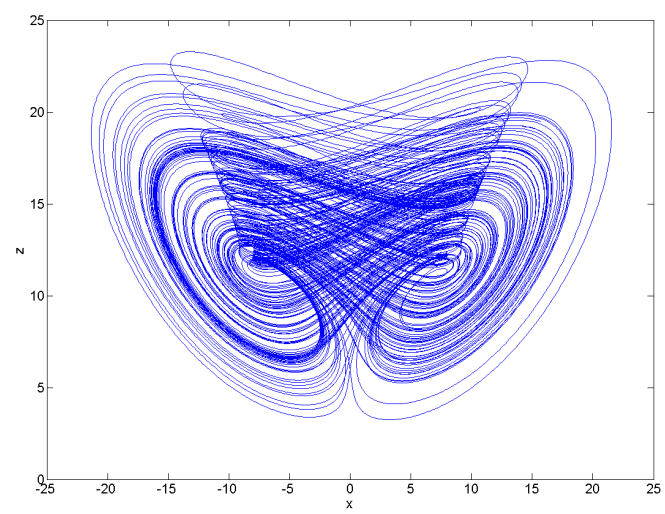

(a)

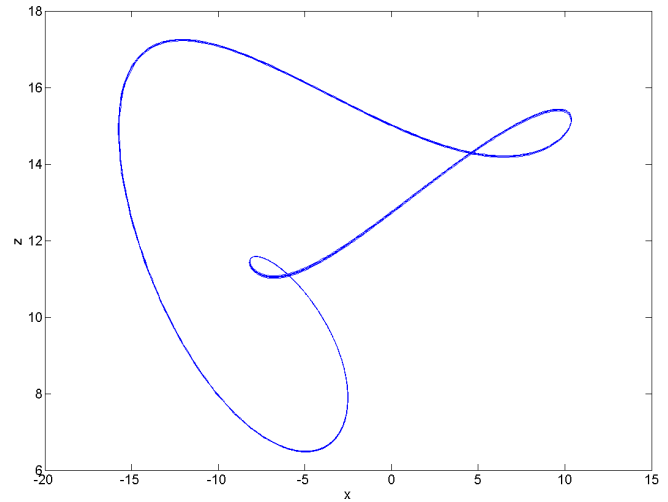

(c)

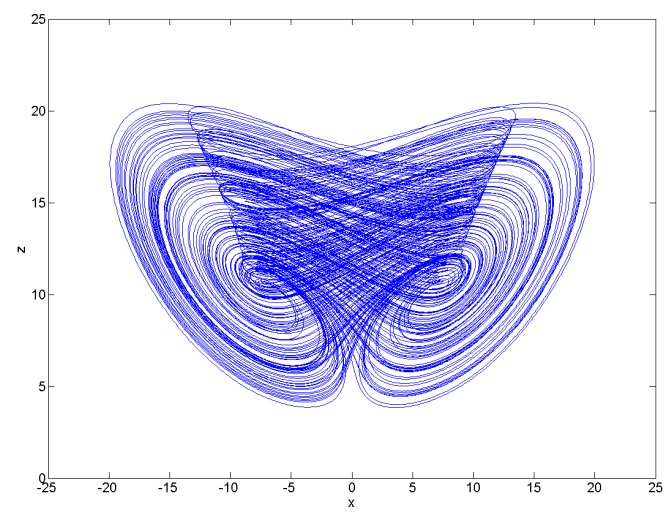

(b)

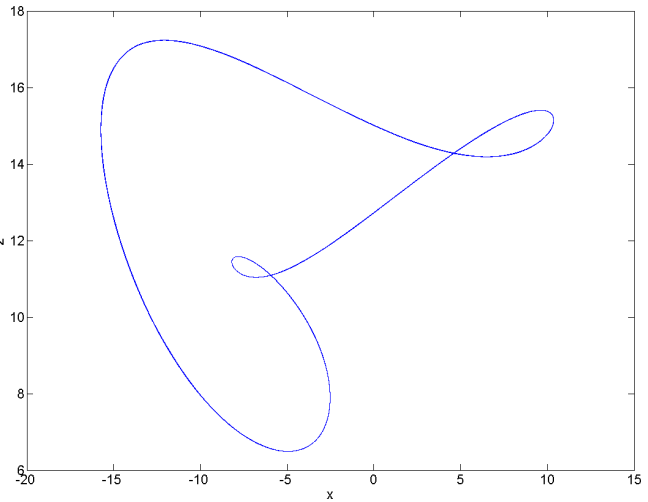

(d)

Figure 4: Phase portrait ( $x$ versus $z$ ) of system (22) with initial value of $x_{0}=1, y_{0}=0.1, z_{0}=0.1$ and (a) $p=56$; (b) $p=58$; (c) $p=p_{5}(t)$; (d) $p=q_{5}=57$. 


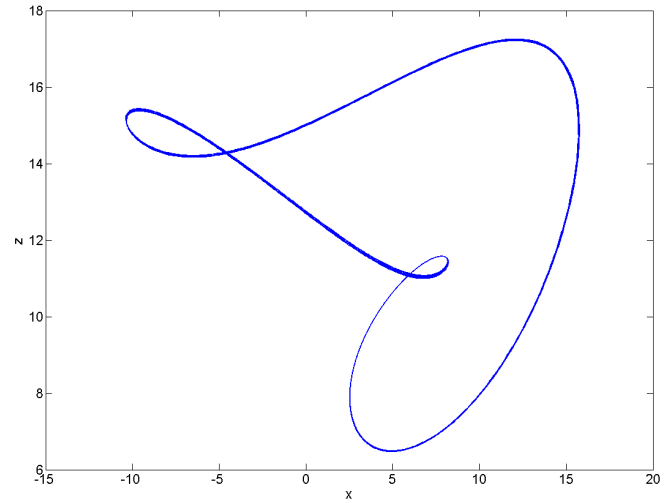

(a)

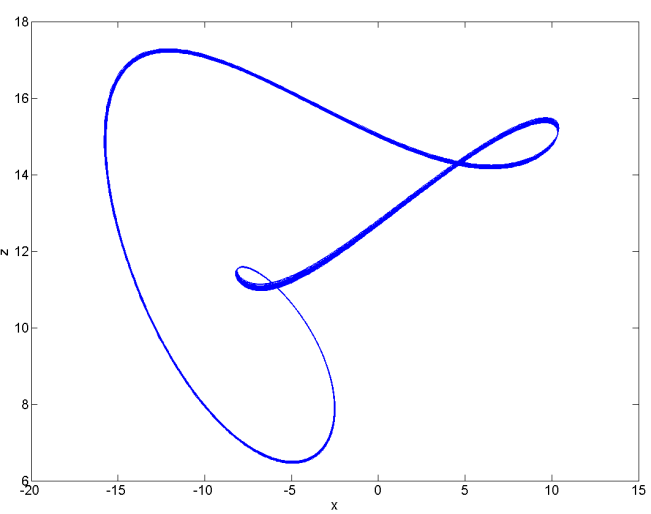

(b)

Figure 5: Phase portrait ( $x$ versus $z$ ) of system (20) with initial value of $x_{0}=10, y_{0}=9, z_{0}=12$ and $p=p_{5}(t)$ when (a) $T=0.01$ and (b) $T=0.005$. 